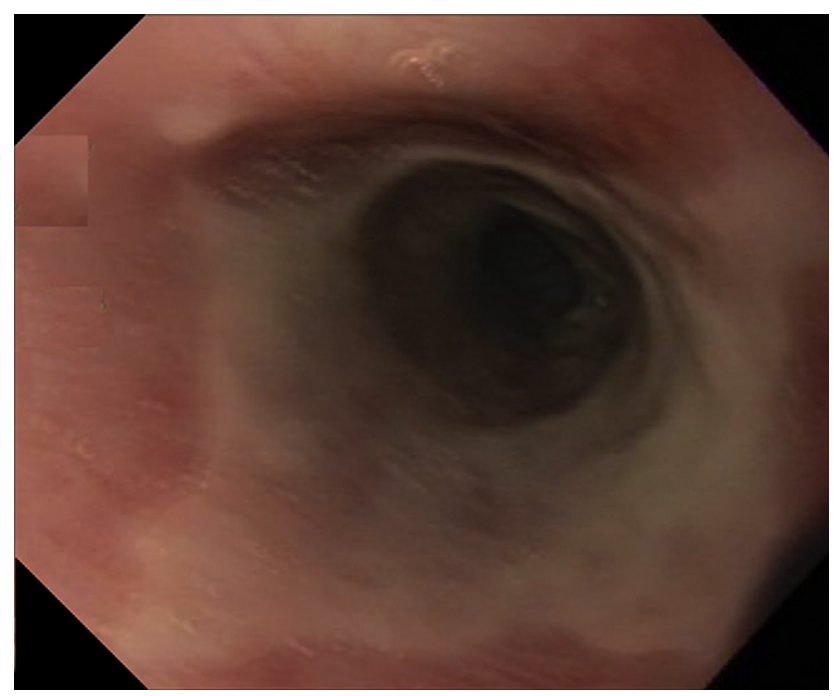

FIGURE 2. Endoscopic view of distal esophagus 4 days after surgical correction of hiatal hernia.

esophageal strictures develop as a late complication. The vast majority of patients with AEN, however, recover fully with supportive care. ${ }^{3}$

In previously reported cases of AEN associated with a hiatal hernia, some authors have performed an esophagectomy. This was done in cases of full-thickness injury or intraoperative perforation. ${ }^{4}$ In other cases, efforts were made to decompress the stomach to allow esophageal healing to take place before proceeding to the operating room. ${ }^{5}$
In our case, the patient's clinical stability suggested that esophageal perforation had not occurred, and we did not consider ourselves obliged to perform an emergency operation. The period of observation also provided reassurance that an esophagectomy would not be necessary in this case and that the patient would be adequately treated with repair of the hiatal hernia. We performed a surveillance endoscopy on postoperative day 4 , as reported in approximately $50 \%$ of cases in the literature. ${ }^{3}$ Observation of mucosal healing, which can occur as early as day 7, may provide some assurance that it is safe to resume oral intake.

We believe that in this rare condition the clinical presentation will dictate the urgency of surgical management. Patients with mediastinitis or sepsis may require an emergency esophagectomy. Conversely, patients in clinically stable condition should be initially observed while treating with an empiric antibiotic course, because partial-thickness AEN typically heals with expectant management. ${ }^{2,3}$

\section{References}

1. Goldenberg SP, Wain SL, Marignani P. Acute necrotizing esophagitis. Gastroenterology. 1990;98:493-6.

2. Ben Soussan E, Savoye G, Hochain P, Hervé S, Antonietti M, Lemoine F, et al. Acute esophageal necrosis: a 1-year prospective study. Gastrointest Endosc. 2002;56:213-7.

3. Gurvits GE. Black esophagus: acute esophageal necrosis syndrome. World $J$ Gastroenterol. 2010;16:3219-25.

4. Kram M, Gorenstein L, Eisen D, Cohen D. Acute esophageal necrosis associated with gastric volvulus. Gastrointest Endosc. 2000;51:610-2.

5. Hwang J, Weigel TL. Acute esophageal necrosis: "black esophagus" JSLS. 2007; $11: 165-7$.

\title{
Assessment by transesophageal echocardiography of left subclavian artery stenosis in patients undergoing coronary artery bypass grafting
}

\author{
Kazumasa Orihashi, MD, Katsuhiko Imai, MD, and Taijiro Sueda, MD, Hiroshima, Japan
}

Although left internal thoracic artery (ITA) is most commonly used in coronary artery bypass grafting (CABG) because of its excellent long-term patency, this benefit can be

\footnotetext{
From the Department of Cardiovascular Surgery, Hiroshima University Hospital, Hiroshima, Japan.

Disclosures: Authors have nothing to disclose with regard to commercial support.

Received for publication Sept 22, 2010; revisions received Nov 16, 2010; accepted for publication Dec 6, 2010.

Address for reprints: Kazumasa Orihashi, MD, Department of Cardiovascular Surgery, Hiroshima University Hospital, Kasumi 1-2-3, Minami-ku, Hiroshima, 734-8551 Japan (E-mail: orichan@hiroshima-u.ac.jp).

J Thorac Cardiovasc Surg 2011;141:e24-6

$0022-5223 / \$ 36.00$

Copyright (c) 2011 by The American Association for Thoracic Surgery doi:10.1016/j.jtcvs.2010.12.003
}

decreased in the presence of stenosis or occlusion in the left subclavian artery (LSCA). Park and colleagues ${ }^{1}$ reported an incidence of $3.5 \%$ (10/284), as assessed by computed tomography (CT). Because of our concerns regarding the nephrotoxicity of contrast agent, however, we have used intraoperative transesophageal echocardiography (TEE) to assess the LSCA. We report the feasibility and results of this method.

\section{CLINICAL SUMMARY}

We examined 152 consecutive CABG cases (January 2005-September 2010, 109 male patients, 46-87 years old) in which use of left ITA graft was scheduled and 
TABLE 1. Profiles and details of stenosis in 7 cases

\begin{tabular}{|c|c|c|c|c|c|c|c|c|}
\hline \multirow[b]{2}{*}{ Case } & \multirow[b]{2}{*}{ Age (y) } & \multirow[b]{2}{*}{ Sex } & \multirow[b]{2}{*}{$\begin{array}{l}\text { Location of } \\
\text { stenosis }\end{array}$} & \multirow[b]{2}{*}{ Stenosis $(\%)$} & \multirow[b]{2}{*}{$\begin{array}{l}\text { Systolic pressure differential } \\
\text { (right arm - left arm, } \mathbf{m m ~} \mathrm{Hg} \text { ) }\end{array}$} & \multirow[b]{2}{*}{$\begin{array}{l}\text { Use of } \\
\text { LITA }\end{array}$} & \multicolumn{2}{|c|}{ Creatinine level (mg/dL) } \\
\hline & & & & & & & Preoperative & $\begin{array}{c}\text { Postoperative } \\
\text { maximum }\end{array}$ \\
\hline 1 & 74 & Male & Proximal & $92 \%$ & $\mathrm{NA}^{*}$ & No & 7.53 & NA \\
\hline 2 & 74 & Male & Proximal & $67 \%$ & 12 & Yes & 3.62 & 5.81 \\
\hline 3 & 70 & Female & Middle & $78 \%$ & -6 & Yes & 0.92 & 1.12 \\
\hline 4 & 56 & Female & Proximal & $88 \%$ & 6 & No & 0.77 & 1.54 \\
\hline 5 & 78 & Female & Middle & $70 \%$ & 7 & Yes & 1.10 & 1.76 \\
\hline 6 & 58 & Male & Middle & $75 \%$ & 12 & Yes & 0.91 & 1.33 \\
\hline 7 & 70 & Male & Proximal & $87 \%$ & 19 & No & 0.93 & 1.42 \\
\hline
\end{tabular}

The percentage stenosis was calculated as follows: $[1-$ (cross-sectional area at maximal stenosis)/(cross-sectional area inside media) $\times 100 \%$. LITA, Left internal thoracic artery; $N A$, not available. *Shunt on left arm for hemodialysis.

TEE assessment was performed by a single TEE examiner (K.O.).

The LSCA was visualized after induction of anesthesia as reported previously. ${ }^{2}$ In the $0^{\circ}$ scanning plane, the transducer was withdrawn from the arch level to visualize the proximal portion of the LSCA in short axis. As the transducer was further withdrawn while being rotated counterclockwise, the middle portion was visualized. The long-axis view of the LSCA was depicted in the $90^{\circ}$ plane. The LSCA was assessed from its origin to the orifice of the left ITA with color Doppler imaging (velocity range $10-50 \mathrm{~cm} / \mathrm{s}$ ).

The LSCA could be visualized in 150 of 152 cases $(98.7 \%)$ ). Apparent stenosis (color flow area $<50 \%$ of vas- cular lumen) was found in 7 cases $(4.7 \%)$ in the proximal portion ( 4 cases) or middle portion ( 3 cases; Table 1$)$. Figure 1 shows the TEE and CT images of concentric stenosis in the proximal LSCA accompanied by calcification in case 7 and eccentric stenosis in the middle portion of LSCA in case 6. Severity of stenosis was assessed with graphics software (CANVAS version 11; ACD Systems International Inc, Victoria, BC, Canada) and calculated as percentage stenosis as follows: [1 - (cross-sectional area at maximal stenosis)/(cross-sectional area inside media) $\times 100 \%$. The LSCA lumen was delineated by serial visualization of LSCA. The percentage stenosis ranged from 0.67 to 0.92 . In 3 patients $(2.0 \%)$ with marked stenosis (percentage stenosis $>80 \%$ ), the right ITA ( 2 cases) or

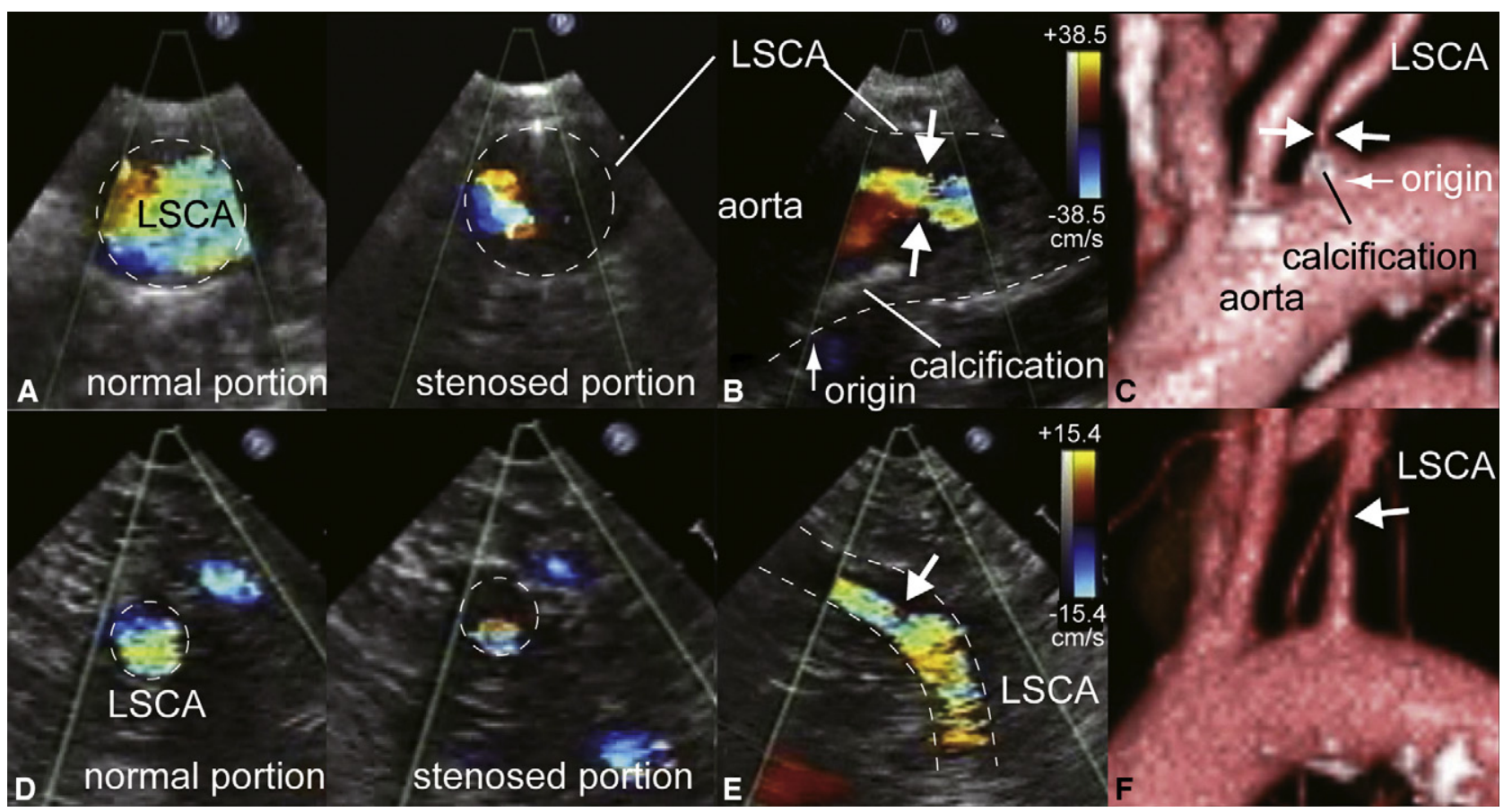

FIGURE 1. Transesophageal echocardiograms and computed tomograms in cases $7(A$ to $C)$ and $6(D$ to $F)$. Severe stenosis with calcification is noted in proximal portion of left subclavian artery $(L S C A)$ in case 7. In case 6, mild stenosis is present in middle portion of left subclavian artery. Velocity range is $38.5 \mathrm{~cm} / \mathrm{s}$ in $\mathrm{A}$ and $\mathrm{B}$ and $15.4 \mathrm{~cm} / \mathrm{s}$ in D and $\mathrm{E}$. 
saphenous vein ( 1 case) was used instead of left ITA. In the other 4 cases with milder stenosis, the left ITA graft was used. Postoperative course was uneventful in all 7 cases. Although the serum creatinine level was elevated postoperatively, it returned to the previous level, and new onset of hemodialysis was not needed. These patients have been carefully followed up.

\section{DISCUSSION}

LSCA stenosis may be overlooked in coronary angiography, because a guidewire can easily enter the LSCA unless it is occluded. The pressure gradient between the bilateral arms is not always predictive of the presence of stenosis. If the left ITA is anastomosed with undiagnosed LSCA stenosis, subclavian steal syndrome may develop and potentially cause angina or life-threatening arrhythmia. ${ }^{3}$

The LSCA can be assessed by $\mathrm{CT}^{1}$ or aortography in coronary angiography. Patients undergoing CABG not uncommonly are seen with abnormal serum creatinine level preoperatively and transient elevation in the perioperative period (Table 1). Contrast agent may be necessitated for other arteriosclerosis-related diseases in the subsequent course. To minimize the need for hemodialysis, it may be advisable to use another modality if available.

We have used intraoperative TEE for assessing the LSCA as well as the aorta (from ascending to abdominal aorta ${ }^{4}$ ), because it occasions no additional cost and is capable of assessing the stenosis without radiation exposure or contrast agent. The LSCA can be visualized in nearly all patients with image resolution comparable to that of $\mathrm{CT}$ imaging. When TEE visualization is difficult, direct echocardiography may be used. Although spectral Doppler assessment may be used for assessing severity, the color Doppler method appears to be sufficient to detect severe stenosis.

Unfortunately, we could not perform a comparative study between the TEE and CT assessment methods, because we had this strategy and the capability of CT was not adequate when we started TEE assessment. At this time, our technique is directed toward detection of severe stenosis but not classification of stenoses into grades. Although the TEE technique appears to require some expertise, an increasing number of anesthesiologists have mastered this technique in many institutions, including ours. No intraobserver or interobserver variability was assessed in this case series. Further investigations with multiple operators and a larger population are mandatory.

In conclusion, intraoperative TEE may be applicable to assessment of the LSCA in cases of CABG with the left ITA graft. It compensates for some drawbacks of radiologic assessments.

\section{References}

1. Park KH, Lee HY, Lim C, Chung ES, Sung SW, Choi S, et al. Clinical impact of computerised tomographic angiography performed for preoperative evaluation before coronary artery bypass grafting. Eur J Cardiothorac Surg. 2010;37:1346-52.

2. Orihashi K, Matsuura Y, Sueda T, Watari M, Okada K, Sugawara Y, et al. Aortic arch branches are no longer blind zone for transesophageal echocardiography: a new eye for aortic surgeons. J Thorac Cardiovasc Surg. 2000;120:466-72.

3. Kursaklioglu H, Kose S, Iyisoy A, Amasyali B, Celik T, Aytemir K, et al. Coronary subclavian steal syndrome presenting with ventricular tachycardia. Yonsei Med J. 2009;50:852-5.

4. Orihashi K, Matsuura Y, Sueda T, Shikata H, Morita S, Hirai S, et al. Abdominal aorta and visceral arteries visualized with transesophageal echocardiography during operations on the aorta. J Thoracic Cardiovasc Surg. 1998;115:945-7. 